\title{
El proceso de valorización de empresas
}

\author{
Magaly Emperatriz Caruajulca Sánchez
}

Ingeniería Industrial n. 27, 2009, ISSN 1025-9929, pp. 169-181

\begin{abstract}
RESUMEN: Las fusiones y adquisiciones son uno de los caminos para el crecimiento de las empresas en la época de globalización de los mercados, y presupone la unificación de capitales ya existentes bajo una dirección unificada. En el mundo se han dado casos importantes de adquisiciones, como el de Nabisco en 1989, el de Warner Communications en 1990, y el de Kraft en 1984. En el Perú también se han dado fusiones y adquisiciones como las de Nicolini por Consorcio Fabril Pacífico, D'Onofrio por el Grupo Gloria, y Solgas por Repsol. En este artículo repasaremos de qué manera se determina el valor de una empresa para su posterior fusión o venta, y se explicará el método que parte del valor contable de la empresa al que se le agrega el valor de los flujos futuros que los activos operativos pueden generar.
\end{abstract}

Palabras clave: globalización de mercados / fusiones y adquisiciones / valorización empresarial / valorización de flujos futuros

\section{The valorization process of business}

ABSTRACT: The globalization of the markets pushes the companies to look for new products, new technologies and new markets. The fusions and acquisitions are one of the ways for the growth of these companies and estimate the unification of already existing capitals under a unified direction.In the world, important cases of acquisitions have been among others, Nabisco in 1989, Warner Communications in 1990, Kraft that were acquired in 1984. In Peru, fusions and acquisitions in different sectors have occurred, between the main ones we counted Nicolini by Manufacturing Partnership Pacific, D'Onofrio by Gloria and Repsol that bought Solgas. How one determines or it calculates the value of a company is what we will develop here. We will explain a method based on the equity and it adds the future flows that the operative assets can generate.

Keywords: globalization of the markets / fusions and acquisitions / corporate valorization / valorization of future flows 


\section{1. ¿QUÉ IMPLICA VALORAR UNA EMPRESA?}

Valorar una empresa, más que asignar un precio de mercado, es una opinión, un proceso mediante el cual se busca cuantificar los elementos que constituyen el patrimonio de una empresa, su actividad, su potencialidad o cualquier característica susceptible de ser valorada. ${ }^{1}$

Esto es importante porque la calidad de las decisiones y de la administración de los recursos de una empresa se miden en términos monetarios. Es decir, si "las matemáticas son el idioma con el que Dios creó el universo" (frase atribuida a Pitágoras), en materia de negocios se puede decir que "[...] el valor monetario es el idioma con el que se mide la creación de valor y el valor mismo de una empresa". ${ }^{2} \mathrm{Si}$ un gerente o empresario considera que la estrategia que aplica en su empresa es efectiva, debe esperar que esta incremente el valor del patrimonio de sus accionistas; lo mismo ocurre cuando se considera correcto usar una tecnología productiva o concentrarse en ciertos mercados.

Hasta aquí, sin embargo, no hemos mencionado un tema crítico en la valorización de empresas: los beneficios futuros, que, al igual que codiciados jugadores de fútbol, valen por lo que generarán en adelante. Para determinar el valor justo de algo es necesario estimar qué beneficios nos deparará en el futuro. Las cosas, en general, no valen per se sino por su capacidad de mejorar la condición de las personas que las poseerán.

\section{2. ¿POR QUÉ VALORIZAR UNA EMPRESA?}

Los motivos pueden ser internos o externos. Los primeros, como su nombre lo indica, están dirigidos al control interno, a los gestores de la empresa y no a determinar su valor para una posterior venta. Los objetivos de estas valoraciones pueden ser:

- Conocer la situación real del patrimonio.

- Verificar la gestión llevada a cabo por los directivos.

- Estudiar la capacidad de deuda.

- Motivos de herencia, sucesión, etcétera.

1 Brugger Jacob, Samuel Inmmanuel. "Valoración de empresas" [en línea]. <http:// www.monografías.com/>.

2 Bravo Orellana, Sergio. Fundamentos de la valorización de empresas, 2002.

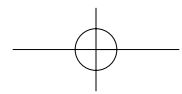


Las valoraciones externas sirven para comprobar y demostrar el valor de la empresa ante terceros, en situaciones como las siguientes:

- Comprar acciones de una empresa.

- Apalancarse mediante emisión de bonos.

- Fusionar dos empresas.

- Adquirir una empresa.

- Reestructurar la organización.

- Negociar la salida de uno de los socios.

En esta tarea la contabilidad no es válida para determinar el "verdadero valor" de una empresa, por cuanto su criterio de valorización de activos muchas veces difiere del valor económico del recurso disponible y, en general, no contempla en forma explícita la capacidad de generación de beneficios futuros. ${ }^{3}$

En forma más específica, la contabilidad no incorpora en los estados financieros aspectos esenciales que agregan valor para el accionista, tales como:

- Capacidad de gestión del equipo gerencial.

- Experiencia de la fuerza laboral.

- Posicionamiento de la empresa en el mercado.

- Sinergia desarrollada por el negocio.

- Valor de una marca, entre otros.

Concluimos entonces que para determinar el valor de una empresa es necesario obtener el valor de los beneficios futuros que el negocio es capaz de proporcionar, considerando la combinación óptima de activos, recursos y habilidades humanas.

\subsection{Métodos de valorización de empresas}

Existen diversos métodos para la valorización de empresas, siendo los más comunes:

3. Atanovski, Dean. "Valorización de empresas". Ponencia en el Seminario organizado por la Asociación de Graduados de ESAN, junio 2009. 
Magaly Caruajulca Sánchez

- Métodos clásicos

- Valor sustancial (1920)

- Según beneficios futuros descontados (1940)

- Métodos mixtos

- Valor medio (1940)

- Métodos nuevos.- Existen diversos métodos basados en la proyección de beneficios futuros.

\subsubsection{Métodos clásicos de valorización}

- Valor sustancial.- Corresponde al valor real de los medios de producción, independientemente de la forma en que estén financiados; es decir, estaría constituido por el valor real de todos los bienes y derechos utilizados. No se considera el valor de los activos no operativos (aquellos no necesarios para la actividad de la empresa) ni la estructura financiera de la empresa.

Normalmente se considera el valor sustancial como el valor mínimo de una empresa:

\begin{tabular}{llll}
\hline Balance general & & & \\
\hline Activos circulantes & 200 & Pasivos & 250 \\
Activos fijos & 300 & Patrimonio (capital contable) & 250 \\
Total activos & 500 & Total pasivo y patrimonio & 500 \\
\hline
\end{tabular}

\section{Entonces:}

Valor sustancial bruto (total de capital) $=$ activos operativos totales $=500$ Valor sustancial neto (capital contable) $=$ activos operativos totales - pasivos $=250$

- Beneficios descontados.- Se orienta del lado de la utilidad, es decir de las ganancias o flujos de caja esperados en el futuro y no del valor actual de la empresa como lo hace el método sustancial. Por consiguiente, el valor depende de los beneficios futuros, del horizonte temporal de valoración y de la tasa de riesgo con la cual se descuenta. El método puede ser muy detallado o general; se pueden crear escenarios futuros o suponer que este será igual que el pasado. La mayoría de las veces se hace un pronóstico muy detallado 
sobre la base de datos pasados para los tres a cinco primeros años y luego se estiman beneficios a perpetuidad.

El peligro es crear escenarios sobre-optimistas o pesimistas que conducen a resultados erróneos.

El valor de la empresa se calcula como:

$$
\mathrm{VE}=\mathrm{U} / \mathrm{r}
$$

Donde:

VE : Valor de la empresa.

$\mathrm{U}$ : Utilidades de la empresa.

$\mathrm{r}$ : Interés que los empresarios esperan de este tipo de negocio.

Si la empresa tiene un crecimiento constante, la fórmula cambia a:

$$
\mathrm{VE}=\mathrm{U} /(\mathrm{r}-\mathrm{g})
$$

Donde:

g : Crecimiento de beneficios constante.

\subsubsection{Métodos mixtos}

- Valor medio

$$
\mathrm{VE}=(\mathrm{a} * \Sigma \text { Activos }+\mathrm{b} * \mathrm{f}(\mathrm{E}[\text { Utilidades }])) /(\mathrm{a}+\mathrm{b})
$$

El valor medio es una combinación de los dos métodos anteriores:

Supone que el valor sustancial es el valor mínimo de la empresa, lo que se recibiría por los activos si estos se vendieran por separado, y toma el valor de beneficios descontados como un valor máximo.

Generalmente se considera:

Valor medio $=(1 *$ Valor Sustancial $+1 *$ Beneficios descontados $) / 2$

\subsubsection{Métodos nuevos}

Los métodos nuevos consideran a la empresa como un proyecto de inversión; por ello, el valor se determina por los rendimientos que se esperan obtener. Se basan en los siguientes conceptos: 
Magaly Caruajulca Sánchez

- Flujo de fondos.- Conjunto de flujos generados disponibles para remunerar a accionistas y prestamistas, luego de haber hecho las inversiones necesarias.

- Tasa de actualización.- Para poder acumular los flujos de diferentes años, es necesario transformarlos al valor actual.

- Horizonte temporal de la valoración.- Cantidad de periodos (generalmente años) en los que se evalúan los flujos de una empresa. Mientras más periodos se tomen más incierto es el pronóstico de los flujos futuros.

- Valor residual.- Es el valor que genera una empresa más allá del horizonte de evaluación planteado. Supone que los flujos son estables en dicho periodo y, por tanto, se puede calcular una renta perpetua:

$$
V R=\frac{F C_{n} *(1+g)}{k-g}
$$

Donde:

VR : Valor residual.

FC : Flujo que se estima estable más allá del horizonte de evaluación; generalmente es el FC del último periodo del horizonte de evaluación.

G : Crecimiento después del horizonte de evaluación.

K : Tasa de actualización.

\subsection{Proceso de valorización bajo el esquema de beneficios futuros}

En este acápite desarrollaremos un método que condensa varios conceptos explicados líneas arriba.

El proceso de valorización bajo este método, se puede esquematizar en las siguientes tres partes: 
1. Due dilligence del balance de corte El objetivo es revisar la situación de la empresa a la fecha de corte, con cuyos datos se producirá la compra o venta de la empresa.

\section{Due dilligence de la base de} las proyecciones

El objetivo es revisar los estados financieros y estudiar los datos históricos para determinar o estimar los mejores supuestos sobre los cuales se proyectarán los flujos del negocio.

\section{Valor de los beneficios futuros y valorización final de la empresa} El concepto es que no son los activos los que determinan el valor de la empresa, sino que este está dado por el valor adicional que la gerencia genera por explotar los activos y convertirlos en flujos comerciales.

El due dilligence consiste en el análisis exhaustivo de los estados financieros de la empresa para determinar su valor real.

En cada una de dichas partes se determina lo siguiente:
1. Valor sustancial del patrimonio
Previamente se calcula:
El valor contable del patrimonio, que se obtiene del balance general y es la pri- mera aproximación.
El valor patrimonial contable corregido, cuando se ajustan los valores declara- dos en el balance general.
El valor sustancial del patrimonio, cuan- do se elimina el valor de los activos no operativos.

2. Valor agregado económico (VAE)

Se parte del estado de resultados proyectados, para calcular luego los flujos comerciales del negocio.

El valor actual (descontado) de los flujos de caja libre constituye el VAE del negocio.

\section{Valor financiero patrimonial (valor de la empresa)}

Es el valor de la empresa calculado como el valor sustancial (obtenido del balance de corte) + el valor económico agregado (de la proyección de beneficios futuros) + el valor de liquidación de los activos no operativos (obtenido del balance de corte).

\subsubsection{Due Dilligence del balance de corte $\rightarrow$ Valor sustancial del patrimonio}

- El valor contable del patrimonio es una primera aproximación al valor de la empresa y el punto de partida del método por desarrollar: 
Magaly Caruajulca Sánchez

\begin{tabular}{lclc}
\hline & US\$ & & US\$ \\
\hline Caja & 40.000 & Cuentas por pagar & 23.000 \\
Cuentas por co- & 60.000 & Deuda corto plazo & 20.000 \\
brar Inventarios & 20.000 & Deuda largo plazo & 15.000 \\
Activo fijo & 180.000 & & 200.000 \\
(depreciación) & $(18.000)$ & Capital social & 30.000 \\
& & Capital adicional & $(6.000)$ \\
& & Utilidades retenidas & 282.000 \\
\hline
\end{tabular}

Valor contable del patrimonio $=$ Activo - Pasivo $=$ US $\$ 224.000$

- Valor contable corregido (VCc).- Se sabe que las cuentas del balance, activos y pasivos, pueden estar incorrectamente dimensionadas:

El activo puede estar sobredimensionado, en cuyo caso hay que identificar la existencia de rubros cuyos valores son ficticios. Será necesario eliminar los valores que no estén debidamente sustentados redimensionando el activo:

$$
\text { Activo real }=\text { activo }- \text { activo ficticio }
$$

Del mismo modo se puede identificar pasivos ficticios; es decir, derechos de terceros que nunca serán reclamados. En consecuencia, se establecerá un pasivo real:

$$
\text { Pasivo real }=\text { pasivo }- \text { pasivo ficticio }
$$

Una vez redimensionados los activos y pasivos, podemos establecer el valor contable corregido (VCc) del patrimonio:

$$
\mathrm{VCc}=\text { Activo real }- \text { Pasivo real }
$$

- Verificación de los saldos del balance

Los estados financieros deben ser ajustados tomando en consideración: 


\section{CAJA}

- Comprobación de saldos

- Fecha de corte

VALORES

- Valor de mercado

CUENTAS POR COBRAR

- Incobrables

- Identificación de la morosidad

INVENTARIOS

- Valor de inventarios

- Inventario físico (eliminar obsoletos, deteriorados)

- Rotación (eliminar productos de baja rotación)

VALORES DE SUBSIDIARIAS

- Valor de mercado de subsidiarias

ACTIVO FIJO

- Revalorización

- Inventario físico de activo fijo

- Activos fijos en liquidación

\section{CUENTAS POR PAGAR}

- Juicios en proceso de pago a proveedores

- Deudas sobredimensionadas o sin sustento DEUDAS

- Posibilidades de renegociación de pasivos

- Reconocimiento de deuda (juicios pendientes)

- Formas de pago

- Posibilidades de capitalización de deuda

Siguiendo las pautas anteriores, el balance quedaría de la siguiente forma:

\begin{tabular}{lcclcc}
\hline Activo & Inicial (US\$) & Real (US\$) & Pasivo & Inicial (US\$) & Real (US\$) \\
\hline Caja & 40.000 & 40.000 & Cuentas por pagar & 23.000 & 40.000 \\
Cuentas por cobrar & 60.000 & 5.000 & Deuda corto plazo & 20.000 & 40.000 \\
Inventarios & 20.000 & 5.000 & Deuda largo plazo & 15.000 & 41.200 \\
\cline { 4 - 6 } Activo fijo & 180.000 & 172.000 & Capital social & 200.000 & 82.000 \\
(depreciación) & $(18.000)$ & $(20.000)$ & Capital adicional & 30.000 & 4.800 \\
& & & Utilidades retenidas & $(6.000)$ & $(6.000)$ \\
\hline Total activo & 282.000 & \multirow{2}{*}{202.000} & Total pasivo y patrimonio & 282.000 & 202.000 \\
\hline
\end{tabular}

$\mathrm{VCc}=$ Activo real - Pasivo real

$\mathrm{VCc}=202.000-121.200$

$\mathrm{VCc}=\mathrm{US} \$ 80.800$

Es importante saber que las correcciones hechas para determinar el VCc producirán pérdidas netas y, por consiguiente, habrá un efecto tributario. 
- Valor sustancial del patrimonio.- Resulta de eliminar del valor contable corregido (VCc), el valor de los activos no ligados a las operaciones del negocio evaluado, considerando solo los bienes y derechos indispensables para la operación.

Los activos no operativos son eliminados al iniciar el proceso de valorización, pero su valor total debe agregarse al final del proceso de valorización. Activos no necesarios para la operación son, por ejemplo, terrenos no edificados, activos de otras actividades, acciones en otros negocios, etcétera.

Si en el ejemplo suponemos que existen US $\$ 30.000$ de activos no operativos, el valor sustancial patrimonial sería:

\begin{tabular}{lclc}
\hline & & Deuda & 121.200 \\
\cline { 3 - 4 } Activo corregido & 172.000 & $\begin{array}{l}\text { Valor sustancial } \\
\text { patrimonial }\end{array}$ & 50.800 \\
& & & \\
\hline $\begin{array}{l}\text { Activo no } \\
\text { operativo }\end{array}$ & $\begin{array}{c}\text { 30,000 } \\
\text { USD }\end{array}$ & $\begin{array}{l}\text { Patrimonio } \\
\text { adicional }\end{array}$ & 30.000 \\
\hline
\end{tabular}

Valor sustancial patrimonial $=\mathrm{VCc}-$ Valor de liquidación de activos no operativos Valor sustancial patrimonial $=$ US $\$ 50.800$

\subsubsection{Due dilligence de la base de proyecciones $\rightarrow$ VAE (valor agregado económico)}

En esta parte se revisa la demanda proyectada, los precios proyectados, los costos y gastos de la operación, el valor de la depreciación y la amortización de activos fijos tangibles e intangibles, el valor residual de dichos activos, las inversiones en bienes de uso operativo, la variación del capital de trabajo, etcétera. En función de esto se proyectarán los flujos de caja libre de la empresa, que son el resultado del accionar del negocio en el tiempo.

El objetivo es determinar el valor agregado económico de dichos flujos y debe incluir el valor agregado adicional que generan las operaciones sobre el valor de los activos (goodwill o plusvalía económica y financiera) o la pérdida de valor respecto a su valor sustancial (badwill o minusvalía económica y financiera). 
Es importante incluir todas las inversiones posibles en la proyección, tanto para el incremento de ingresos (incremento de capacidad y productividad, mejora de calidad, etcétera) como para la reducción de costos operativos, administrativos y financieros. Esto debe tener su correspondencia y coherencia con el flujo de ingresos (ventas) y egresos (gastos) del estado de ganancias y pérdidas (GyP) proyectado. No deben incluirse proyectos con poca probabilidad de ejecutarse o de rentabilidad incierta.

El valor agregado económico se calculará como el valor actual de los flujos de caja libre, considerando que:

Utilidad neta del GyP proyectado

+ Cuentas no monetarias (depreciación, amortización, etcétera)

- Inversiones en bienes de uso operativo (a lo largo del horizonte de evaluación)

+/- Variación del capital de trabajo

+ Valor residual de activos fijos

- Dividendos

- REI (resultado por exposición a la inflación o ajuste por inflación)

\section{= Flujo de caja libre (FCL)}

En nuestro ejemplo encontramos que descontando los flujos de caja libre y considerando cinco años de horizonte de evaluación:

\begin{tabular}{llllll}
\hline Año & 1 & 2 & 3 & 4 & 5 en adelante \\
\hline Monto & $\mathrm{FCL}_{1}$ & $\mathrm{FCL}_{2}$ & $\mathrm{FCL}_{3}$ & $\mathrm{FCL}_{4}$ & $\mathrm{FCL}_{5}$ \\
\hline
\end{tabular}

Para nuestro caso, vamos a considerar que el valor descontado de dichos flujos es:

Valor agregado económico $=$ Valor actual $($ tasa de descuento, FCLi $)$

$\mathrm{VAE}=\mathrm{US} \$ 447,245$ 


\subsubsection{Valor financiero patrimonial (valor de la empresa)}

Partiendo entonces de que el valor de la empresa no está dado por el valor de los activos sino por el valor que estos generan, se concluye que el valor está dado por el valor sustancial (obtenido del balance de corte) + el valor económico agregado (de la proyección de beneficios futuros) + valor de liquidación de los activos no operativos (a la fecha de corte).

\begin{tabular}{lclc}
\hline \multirow{2}{*}{ Activo corregido } & 172.000 & Deuda & 121.200 \\
\cline { 3 - 4 } & & Valor agregado económico & 447.245 \\
\hline $\begin{array}{l}\text { Valor agregado } \\
\text { económico }\end{array}$ & 447.245 & Valor sustancial patrimonial & 50.800 \\
\hline & & & 30.000 \\
\hline Activo no operativo & 30.000 & Patrimonio adicional & 3 \\
\hline
\end{tabular}

Valor empresa $=$ Valor sustancial + Valor agregado económico + Valor liquidac. de activos no operativos

En este caso, el valor se estimaría en:

Valor empresa $=50.800+447.245+30.000$

Hay que notar que se ha tomado el valor de liquidación de los activos no operativos como el valor contable de estos y no siempre es así.

Por último, cabe señalar que no existe un único valor para una empresa, ya que este depende de las expectativas y percepciones de quienes hacen la proyección y la evaluación. 
El proceso de valorización de empresas

\section{BIBLIOGRAFÍA}

Brugger, Samuel Inmmanuel. "Valoración de empresas" [en línea]. $<$ http://www.monografias.com/>.

Bravo, Sergio. Fundamentos de la valorización de empresas. Lima: ESAN, 2002.

Atanovski, Dean. "Valorización de empresas". Seminario organizado por la Asociación de Egresados de ESAN. Lima, junio del 2009.

García, Beatriz e y José Antonio Pérez. "Análisis económico y creación de valor". Finanzas y Contabilidad 61. Madrid: Ediciones Deusto, 2004.

PKF Vila Naranjo y Asociados. Boletín de interpretación contable y tributario 26. Lima, octubre del 2005. 